УДК 628.58

\title{
МОДЕЛИРОВАНИЕ РАСПРОСТРАНЕНИЯ В АТМОСФЕРЕ ЗАГРЯЗНЯЮЩИХ ВЕЩЕСТВ ВЫБРОСОВ ЭЛЕКТРОСТАНЦИЙ НА БАЗЕ ПРОГРАММНОГО КОМПЛЕКСА «ККАТ»
}

\author{
Антонова Александра Михайловна', \\ anton@tpu.ru \\ Воробьев Александр Владимирович', \\ worob@tpu.ru \\ Воробьев Владимир Александрович', \\ worob@tpu.ru \\ Дутова Екатерина Матвеевна', \\ dutova@tpu.ru \\ Покровский Виталий Дмитриевич², \\ vdp@mail.ru \\ ' Национальный исследовательский Томский политехнический университет, \\ Россия, 634050, г. Томск, пр. Ленина, 30 \\ 2 Национальный исследовательский Томский государственный университет, \\ Россия, 634050, г. Томск, пр. Ленина, 36.
}

\begin{abstract}
Актуальность рассматриваемой тематики заключается в необходимости повышения точности прогнозирования содержания загрязняющих веществ в атмосферном воздухе от выбросов объектов энергетики, в том числе атомных станций, при проведении независимой оценки.
\end{abstract}

Цель работы: обоснование выбора методики расчета рассеивания газообразных загрязняющих веществ от непрерывно действующих высоких источников с учетом метеорологических факторов и тестирование опыта эксплуатации программного комплекса.

Объекты: современные методы расчетов рассеивания выбросов загрязняющих веществ в атмосферном воздухе, рекомендуемые для использования на территории России и методика Пасквилла для расчетов загрязнения от непрерывно действующего высокого источника при различных стационарных метеорологических условиях.

Методы: математическое моделирование и расчетный анализ на основе разрабатываемого компьютерного программного комплекса «SKAT».

Результаты. Проведен анализ методов по рассеиванию газообразных загрязняющих веществ в атмосфере: МРР-2017 и Пасквилла. Сделан вывод о том, что для расчетов рассеивания газообразных загрязняющих веществ выброса объектов энергетики для различных категорий состояния атмосферы в условиях постоянства метеорологических факторов предпочтительнее использовать методику Пасквилла, основанную на модели Гаусса. Разработанный программный комплекс «SКАТ» может быть использован для прогнозирования приземных концентраций загрязняющих веществ и расчетов рассеивания загрязняющих веществ в атмосферном воздухе при решении задач мониторинга выбросов загрязняющих веществ в условиях действующих объектов. Применительно к условиям г. Северска Томской области для проектируемого энергоблока с реактором БРЕСТ-300 и пристанционным ядерным топливным циклом по модели Гаусса проведены расчеты распределения выбросов радионуклидов в приземном слое атмосферы.

\section{Ключевые слова:}

Загрязняющие вещества, моделирование, рассеивание, атмосферный воздух турбулентная диффузия, защита окружающей среды.

\section{Введение}

Процессы переноса загрязняющих веществ (3В) от объектов энергетики и промышленности являются существенным фактором, влияющим на социальную обстановку и экономическое состояние регионов мира [1]. Необходимость повышения точности прогнозирования содержания 3В в атмосфере определяет актуальность этих исследований $[2,3]$.

Выбросы ЗВ из труб объектов энергетики при поступлении в атмосферу включаются в многообразные процессы движения атмосферного воздуха, осаждаются на почву, распространяются в гидросфере, загрязняя поверхностные и подземные воды. Исследования закономерностей сложных процес- сов переноса загрязняющих веществ и прогнозирования содержания ЗВ в объектах биосферы в нашей стране [4-8] и за рубежом [9-11] ведутся со второй половины XX в.

Для тепловых электростанций (ТЭС) характерно поступление через дымовую трубу в атмосферу химических загрязнителей в виде газов и аэрозолей, образующихся при сжигании органического топлива. Основные из них - оксиды C, H, O, N, S и микроэлементы (мышьяк, кадмий, никель, кобальт, медь, свинец, ртуть), содержащиеся в угле, значительная часть которых не улавливается фильтрами. Атомные станции (АС) выбрасывают в атмосферу через вентиляционную трубу газоаэро- 
зольную среду, большую часть активности которой составляют радиоактивные благородные газы (РБГ), такие как Ar, Xe, Kr. Кроме того, в состав выброса входят активационные газы $\left({ }^{14} \mathrm{C},{ }^{16} \mathrm{~N}\right)$, paдионуклиды йода и долгоживущие нуклиды в аэрозольной форме $(\mathrm{Co}, \mathrm{Cs})[12,13]$; все составляющие выброса АС строго нормированы.

Соблюдение установленных норм содержания ЗВ в приземном слое атмосферы требует прогнозирования на основе математических моделей с использованием метеорологических данных, адекватно отражающих рассеивание, и последующего контроля содержания ЗВ в атмосферном воздухе. Разработано множество моделей разной степени сложности [14-17], как правило, на практике их использование сопряжено с большими трудностями.

Для высоких источников, каковыми являются дымовые трубы ТЭС и вентиляционные трубы АС, ключевыми функциями рассеивания являются: распределение приземных концентраций ЗВ в направлении оси струи выброса, значения максимальной концентрации, расстояния от источника, на котором они достигаются и опасной скорости ветра. Для одного и того же источника эти функции определяются типом устойчивости атмосферы и скоростью ветра.

\section{Теоретические основы рассеивания примесей в атмосфере}

Газоаэрозольный выброс объектов энергетики имеет теплогидравлический подъем над устьем трубы, потому как температура выброса всегда выше температуры окружающего воздуха. Поэтому взаимодействие выброса с атмосферным воздухом начинается выше устья трубы на высоте $H_{\text {эфф }}$.

Выброс из точки $\left(H_{\text {эфф}}, x\right)$, постепенно удаляясь от трубы, рассеивается вследствие динамической турбулентности, вызванной ветром. При касании факела земли в приземном слое атмосферы появляются составляющие выброса - газы или аэрозоли.

Для решения задачи о распространении газоаэрозольных выбросов надо учитывать строение атмосферы и законы движении воздуха в ней. При этом наибольший интерес представляет ближайший к поверхности земли слой атмосферы - тропосфера. Движение воздуха во всем объеме тропосферы является турбулентным, что приводит к быстрому рассеиванию, при этом главную роль играют градиент ветра и температуры, как в пространстве, так и во времени. Происходит диффузия на фоне основного осредненного движения [18].

Функциональное описание рассеяния радионуклидов в атмосфере предполагает их турбулентное движение в одной плоскости и диффузионное в двух других плоскостях, имеет вид [19]

$$
\frac{\partial a}{\partial \tau}+u \frac{\partial a}{\partial x}+v_{g} \frac{\partial a}{\partial z}=K \nabla^{2} a-\lambda a,
$$

здесь

$$
K \nabla^{2} a=\frac{\partial}{\partial x} K_{x} \frac{\partial a}{\partial x}+\frac{\partial}{\partial y} K_{y} \frac{\partial a}{\partial y}+\frac{\partial}{\partial z} K_{z} \frac{\partial a}{\partial z}
$$

где $a$ - содержание радионуклида; $x, y, z$ - координаты движущейся частицы; $u$ - скорость ветра; $v_{g}-$ скорость гравитационного осаждения; $\lambda$ - постоянная радиоактивного распада; $K$ - коэффициент турбулентности; $K_{x}, K_{y}, K_{z}-$ коэффициенты турбулентной диффузии, характеризующие интенсивность турбулентного обмена;

$$
K_{x}=\frac{1}{2} \frac{d \sigma_{x}^{2}}{d \tau} ; K_{y}=\frac{1}{2} \frac{d \sigma_{y}^{2}}{d \tau} ; K_{z}=\frac{1}{2} \frac{d \sigma_{z}^{2}}{d \tau} .
$$

Здесь $\sigma_{x}, \sigma_{y}, \sigma_{z}$ - квадратичные дисперсии координат движущейся частицы:

$$
\begin{gathered}
\sigma_{x}^{2}(\tau)=[x(\tau)-x(0)]^{2} ; \quad \sigma_{y}^{2}(\tau)=[y(\tau)-y(0)]^{2} ; \\
\sigma_{z}^{2}(\tau)=[z(\tau)-z(0)]^{2} .
\end{gathered}
$$

В уравнение турбулентной диффузии входит слагаемое, содержащее скорость ветра, это принципиально отличает его от уравнения фикковской диффузии и существенно усложняет решение дифференциального уравнения. Коэффициенты турбулентной диффузии имеют значения, в десятки и сотни тысяч раз превышающие значения коэффициентов молекулярной диффузии, они различны в разных направлениях и меняются вдоль каждой координаты (особенно вдоль вертикальной $Z$ ).

Для решения задачи рассеивания загрязняющих веществ в атмосфере с помощью уравнения турбулентной диффузии необходимо знать значения составляющих вектора скорости ветра в зависимости от времени. Поэтому использование этого уравнения дает лишь приближенное решение с высокой степенью погрешности. Кроме того, необходим учет типа подстилающей поверхности (ее шероховатости) [20], вымывающей способности осадков и других факторов.

\section{Анализ методик расчета рассеивания примесей в атмосфере}

В отечественной практике в настоящее время используются в основном две методики, рекомендуемые для расчета загрязнения атмосферного воздуха загрязняющими веществами промышленного происхождения. Это современные методы расчетов рассеивания выбросов загрязняющих веществ в атмосферном воздухе, рекомендуемые для использования на территории России (далее - МРP-2017), и методика, разработанная Ф. Пасквиллом (так называемая Гауссова модель). Всемирная метеорологическая организация предложила использовать указанную методику для расчетов загрязнения атмосферного воздуха от выбросов энергетических и промышленных предприятий.

В основе методики МРP-2017 [3] лежат предположения о влиянии на характер и масштабы рассеивания загрязняющих веществ в приземной части атмосферы как метеорологических параметров (стратификация атмосферы, скорость и направление ветра, температура воздуха), так и параметров источника выброса и состава газоаэрозольной смеси.

Восстановление пространственного распределения концентрации загрязняющих веществ базируется на расчете следующих основных параметров: 
$u_{\mathrm{M}}-$ опасной скорости ветра; $x_{\mathrm{м}}-$ расстояния от источника выброса до точки с максимальной приземной концентрацией; $C_{m}-$ максимального значения приземной концентрации.

Для одиночного точечного источника с круглым устьем максимальная величина приземной разовой концентрации ЗВ от выброса пылегазовоздушной смеси вычисляется по уравнению

$$
C_{\mathrm{m}}=\frac{A M F m n \eta}{H^{2} \sqrt[3]{V_{1} \Delta T}}
$$

где $C_{\mathrm{м}}-$ в мг $/ \mathrm{M}^{3}\left(\mathrm{мБ \kappa} / \mathrm{M}^{3}\right) ; A-$ коэффициент, определяемый типом стратификации атмосферы; $M, V_{1}$ - активность 3В, выбрасываемых в атмосферу в единицу времени, г/с (Бк/с) и объемный расход газоаэрозолей, $\mathrm{m}^{3} ; F, m, n$ - безразмерные коэффициенты, зависящие от скорость оседания ЗВ в атмосферном воздухе и от условий выхода газоаэрозольной смеси из вентиляционной трубы; $\eta$ - безразмерный коэффициент, учитывающий рельеф местности; $H$ - высота источника выброса над уровнем земли, м; $\Delta T=T_{\mathrm{r}}-T_{\text {в }}$ - перепад между температурой выбрасываемой газоаэрозольной смеси $T_{\text {г }}$ и температурой атмосферного воздуха $T_{\mathrm{в}},{ }^{\circ} \mathrm{C}$.

Расстояние от источника выброса до точки с максимальной приземной концентрацией $3 \mathrm{~B}$ рассчитывается по формуле

$$
x_{\mathrm{M}}=\frac{5-F}{4} d H,
$$

где $d$ - безразмерный коэффициент.

Алгоритм определения опасной скорости ветра

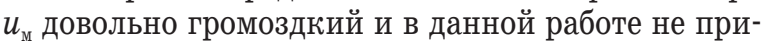
водится.

Методика МРР-2017 рекомендована в настоящее время для проведения расчетов рассеивания выбросов загрязняющих веществ в атмосферном воздухе с целью нормировании и установления ПДВ (предельно допустимых выбросов). Одним из недостатков данной методики считается ограниченность рекомендаций для вычисления рассеяния загрязняющих веществ при задании конкретных метеорологических условий в случае кратковременно действующего источника. Данную методика затруднительно применять для прогнозных расчетов при конкретных метеорологических условиях и специфических источниках выбросов. Кроме этого, методика МРР-2017 не позволяет в полной мере учесть тип подстилающей поверхности.

Методика Пасквилла $[2,21]$ основана на Гауссовой модели рассеивания. При истечении загрязняющих веществ в атмосферу образуется облако, которое сносится вместе с окружающим атмосферным воздухом по направлению ветра. В процессе движения происходит турбулентное перемешивание с окружающим атмосферным воздухом, что приводит к расширению облака в пространстве и изменению концентрации ЗВ в нем. В итоге формируется пространственное распределение концентрации загрязняющих веществ, которое в са- мом общем случае описывается нормальным (Гауссовым) законом.

В соответствии с этой методикой, при неизменных метеорологических условиях диффузия примеси от непрерывного источника приводит к приземной концентрации ЗВ, которую можно найти с помощью выражения:

$$
C(x)=\frac{Q}{\pi \sigma_{y} \sigma_{z} u} \exp \left[-\left(\frac{H_{\text {эфф }}^{2}}{2 \sigma_{z}^{2}}\right)\right] f_{F} f_{W},
$$

где $C(x)$ - концентрация (объёмная активность), Бк $/ \mathrm{M}^{3} ; Q$ - мощность источника, Бк/с; $H_{\text {эфф }}$ - эффективная высота выброса, м; $u$ - скорость ветра на эффективной высоте выброса, $\mathrm{M} / \mathrm{c} ; \sigma_{y}, \sigma_{z}-$ коэффициенты дисперсии распределения Гаусса (турбулентной диффузии) в направлениях, перпендикулярных к направлению ветра, м; $f_{f}$ - эмпирический фактор обеднения, учитывающий сухое осаждение; $f_{W}$ - эмпирический фактор обеднения, учитывающий вымывание осадками.

Для расчёта коэффициентов вертикальной $\sigma_{z}$ и горизонтальной $\sigma_{y}$ турбулентной диффузии используются эмпирические формулы Смита-Хоскеpa [19], считающиеся в настоящее время достаточно надежными. Указанные коэффициенты включают в себя факторы, зависящие от состояния устойчивости атмосферы и типа подстилающей поверхности.

К основным достоинствам модели Гаусса относится возможность учесть влияние на распределение загрязняющего вещества таких важных факторов, как:

- состояние устойчивости атмосферы;

- тип (шероховатость) подстилающей поверхности;

- тепловой подъем струи вследствие перегрева выбрасываемой газоаэрозольной смеси;

- выпадение примесей на поверхность земли;

- вымывающая способность осадков и др.

K недостаткам Гауссовой модели можно отнести ограничения использования по скорости ветра (модель нельзя использовать при $u<1 \mathrm{~m} / \mathrm{c}$ ) и расстояниям от источника (модель работает до 10 км).

Гауссова модель рассеивания примеси лежит в основе методик МАГАТЭ, которые включают рекомендации для вычисления дисперсий по исходным параметрам состояния атмосферы. Эти разработки, основанные на результатах экспериментальных исследований Ф. Пасквилла и Ф. Гиффорда, используются для широкого спектра расчетов зарубежных коллег.

\section{Моделирование поля концентраций загрязняющих веществ от выбросов энергообъектов}

Цель исследования - установить вид распределения в приземном слое атмосферы концентраций 3В газоаэрозольного выброса энергообъектов (тепловой или атомной станции). Исследованию подлежит поведение выброса в атмосферном воздухе при различных сочетаниях влияющих факторов - 
скорости ветра, категории атмосферы по Пасквиллу, вида осадков, значения температуры выброса.

Ставятся задачи:

- определения значений приземной концентрации основных ЗВ выброса в зависимости от расстояния от точки выброса;

- определения влияния различных факторов на поведение факела выброса в атмосферном воздухе;

- построения графических зависимостей концентрации радионуклидов от расстояния от источника выброса.

Для решения задач использованы методики расчета концентраций МРР-2017 и Пасквилла.

Принято, что скорость ветра меняется по высоте и направлению, поскольку, по мере удаления от источника, увеличивается вероятность изменения направления ветра. Учтено влияние рельефа местности, высоких препятствий, водоемов на распространение загрязняющих веществ. С помощью факторов истощения учтены такие явления, как снижение активности вследствие естественного распада, вымывание газовой примеси в подоблачном слое, химические реакции между примесью и атмосферным воздухом.

При моделировании приняты следующие допущения.

1. Дисперсия концентрации радионуклидов в направлении, поперечном к оси факела, рассчитывается по упрощенной формуле.

2. Приняты среднегодовые значения скорости ветра.
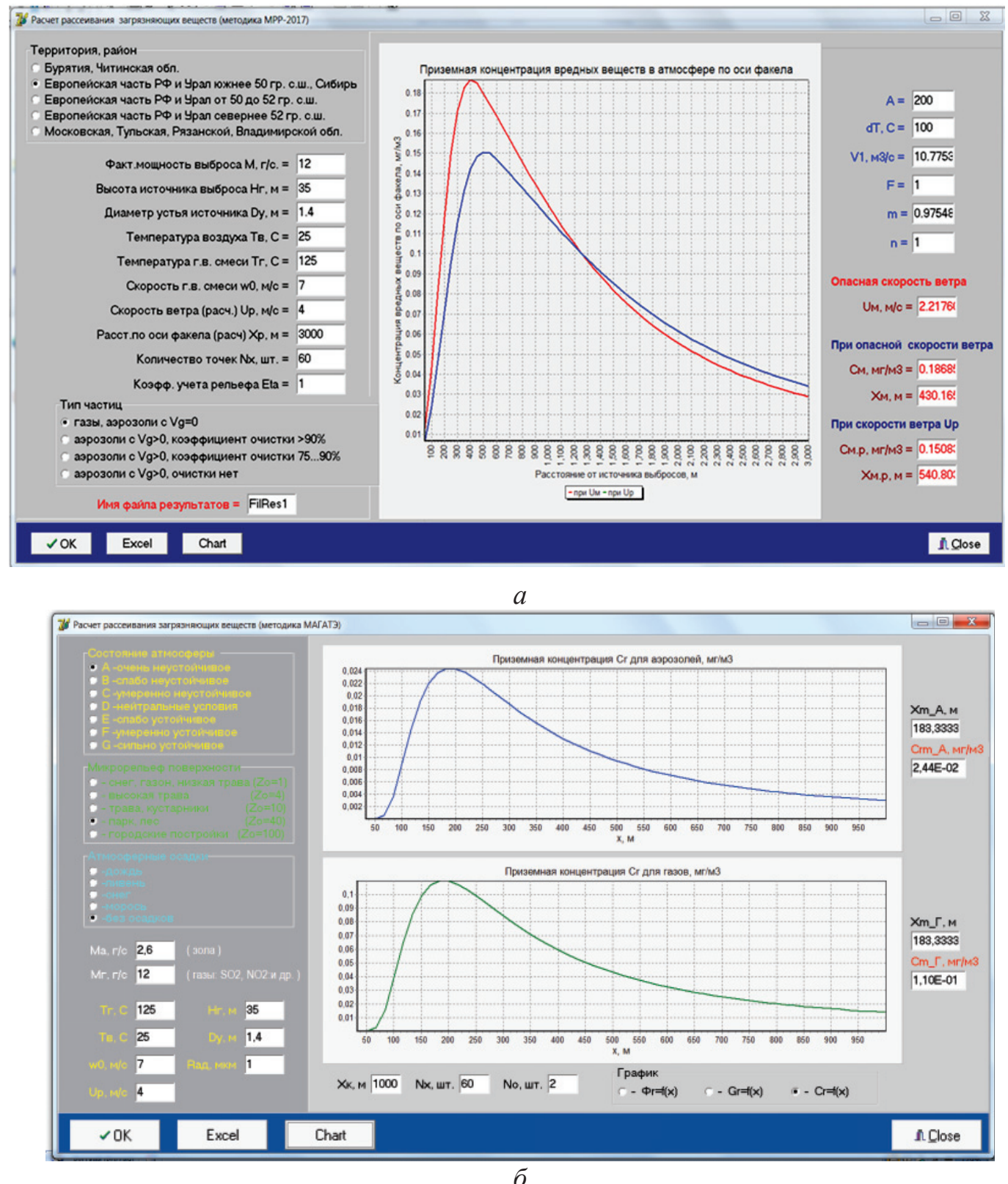

Puc.1. Скриншот главного окна исполняемого модуля «Scat_MRR» (а) и главного окна исполняемого модуля «Scat_PSC» (б)

Fig. 1. Screenshot of the main window of the Scat_OND executable (a) and the main window of the Scat_PSC executable (б) 
3. Не учитываются фоновые значения концентраций загрязняющих веществ.

Несмотря на перечисленные выше упрощения, с помощью разработанной модели оказалось возможным произвести достоверную количественную оценку поля концентрации от выброса загрязняющих веществ.

\section{Описание программного комплекса}

Для реализации алгоритмов расчета рассеивания ЗВ в соответствии с рассмотренными выше методиками создан программный комплекс «SKAT».

Комплекс, разработанный в лицензионной системе программирования Borland Turbo Delphi 7, представляет собой приложение, функционирующее в операционной среде MS Windows и совместимых с ними ПЭВМ. Текущая версия программного комплекса «SKAT» включает 2 специализированных исполняемых модуля: «Scat_PSC》 и «Scat MRR».

В исполняемых модулях комплекса имеется возможность графической интерпретации результатов расчета и импорта последних в MS Excel. Скриншоты главных окон исполняемых модулей программного комплекса «SKAT» представлены на рис. 1.

\section{Результаты расчетов}

С помощью разработанного программного комплекса «SCAT» были выполнены сравнительные расчеты рассеивания газообразных загрязняющих веществ по двум методикам: Пасквилла $[2,21]$ и МРP-2017 [3]. В качестве исследуемого объекта рассматривались непрерывно действующий источник выброса дымовых газов $\left(\mathrm{SO}_{2}, \mathrm{NO}_{2}\right)$.

В расчетах приняты следующие значения входных данных:

- мощность источника выброса по газам $\left(M_{\mathrm{r}}\right)$ и по аэрозолям $\left(M_{a}\right)$ равна 12 и 2,6 г $/ \mathrm{c}$, соответственно;

- скорость газоаэрозольной смеси в устье источника выброса $\left(w_{0}\right)$ равна 7 м $/$;

- высота выброса смеси $(H)$ равна 35 м;

- диаметр устьевой части источника $\left(D_{y}\right)$ равен $1,4 \mathrm{~m}$;

- диапазон изменения температур окружающего воздуха и выбрасываемой смеси $(\Delta T)$ равен $100{ }^{\circ} \mathrm{C}$;

- скорость ветра на высоте выброса опасная (в 2,1 и $2,25 \mathrm{~m} / \mathrm{c})$.

Перечисленные выше параметры соответствуют известному из [1] тестовому расчету.

Результаты расчетов по сравниваемым методикам представлены на рис. 2-4.

Анализ содержимого рис. 2 показывает, что обе указанные выше методики дают близкие оценки значений опасной скорости ветра, в 2,1 и 2,25 м/с по методике МРP-2017 и Пасквилла, соответственно, при которой возможно формирование максимального содержания загрязняющих веществ.

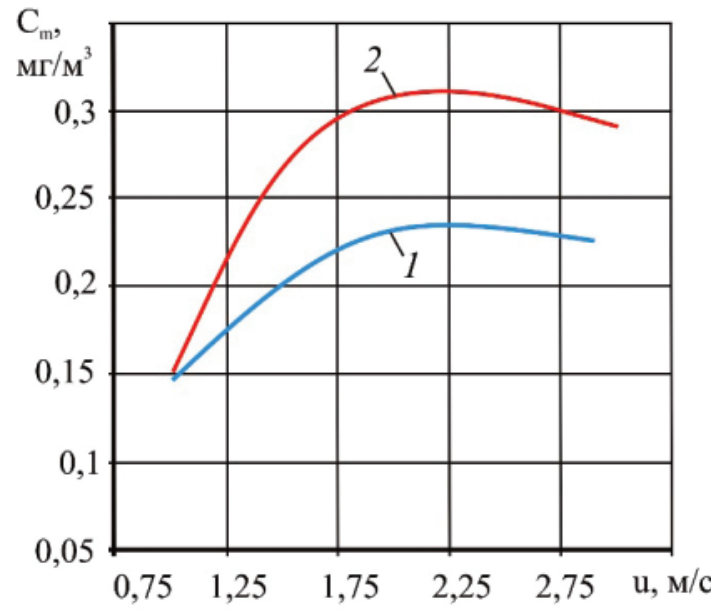

Pис. 2. Влияние скорости ветра на характер изленения максимальной кониентрации $\mathrm{SO}_{2}, \mathrm{NO}_{2}$ по оси факела в случае неблагоприятных метеорологических условий. Расчет по методике: 1 - МРР-2017; 2 - Пасквилла

Fig. 2. Change in the maximum concentration along the axis of the torch depending on the wind speed in case of adverse meteorological conditions. Calculation according to the: 1 - MRR2017 method; 2 - Pasquill method

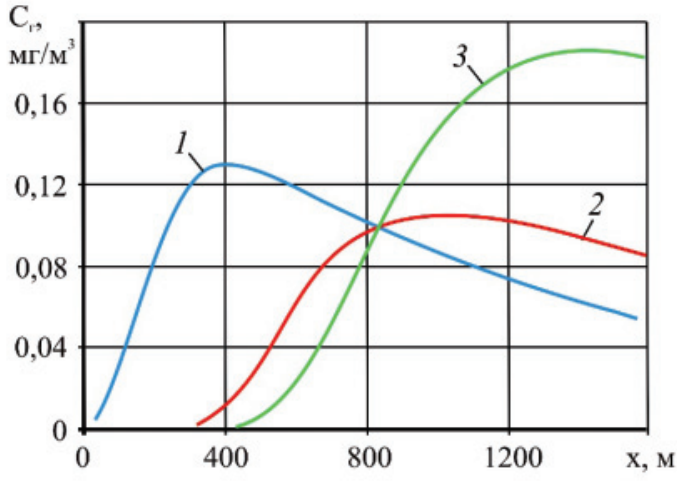

$a$

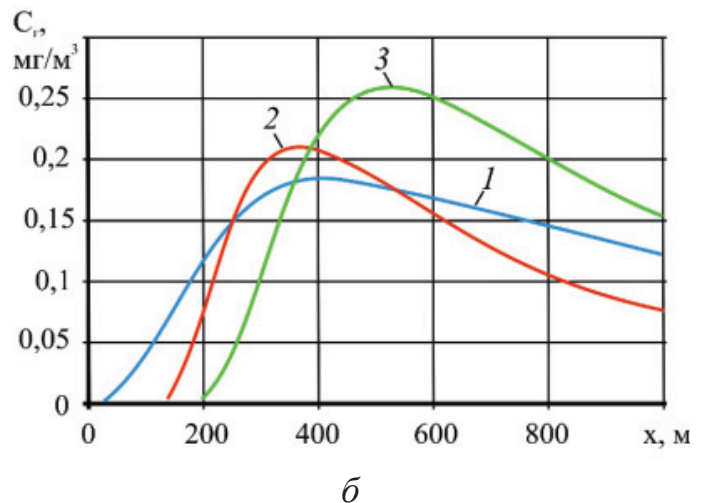

Puс. 3. Распределение приземной концентрации $\mathrm{SO}_{2}, \mathrm{NO}_{2}$ вдоль оси факела при опасной скорости ветра, соответствующее благоприятным (а) и неблагоприятныл (б) метеорологическим условиям: 2 и 3 - расчет по методике Пасквилла ( $z_{0}=10$ и 1 слм); 1 - расчет по методике МРP-2017

Fig. 3. Distribution of $\mathrm{SO}_{2}, \mathrm{NO}_{2}$ surface concentration along the axis of the torch at dangerous wind speed, corresponding to favorable (a) and unfavorable (б) meteorological conditions: 2 and 3 - calculation using the Pasquill method $\left(z_{0}=10\right.$ and $1 \mathrm{~cm}) ; 1$ - calculation according to the MMP-2017 method 
При благоприятных метеорологических условиях (рис. $3, a$ ) результаты моделирования распределения концентрации газообразных загрязняющих веществ по оси факела выброса существенным образом зависят от выбранной методики расчетов (МРР-2017 или Пасквилла). Причем положение точки с максимальным значением приземной концентрации, найденное по формулам MRR-2017, практически не зависит от метеорологических параметров, что противоречит здравому смыслу и данным [10, 15].

Напротив, при неблагоприятных метеорологических условиях (рис. 3 , б) распределение концентрации ЗВ мало зависит от использованной методики расчета, в первую очередь это касается расположения точки с максимальным значением приземной концентрации ЗВ.

Анализ кривых на рис. 3 , рассчитанных с использованием методики Пасквилла, позволяет сделать вывод о существенной зависимости положения максимума приземной концентрации от шероховатости подстилающей поверхности: изменение шероховатости подстилающей поверхности с 1 до 100 см приводит к смещению расположения точки с максимальной концентрацией с 1400 до 660 м. Менее заметно влияние шероховатости на величину самого максимума. В методике МРP-2017 учесть тип подстилающей поверхности затруднительно.

На рис. 4 приведены результаты расчета мак-

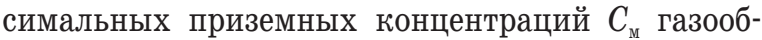
разных ЗВ и расстояний $x_{\mathrm{m}}$ от источника выброса, где при различных метеорологических условиях эти значения концентраций достигаются.

По модели Гаусса проведены вариантные расчеты распределения концентраций радионуклидов выброса в приземном слое атмосферы для условий г. Северска Томской области для проектируемого энергоблока АC с реактором БРЕСТ-300 с пристанционным ядерным топливным циклом.

В этом исследовании выполнен комплекс расчетов содержаний РБГ, радионуклидов йода и аэрозолей в приземном слое атмосферы при нормальной работе $\mathrm{AC}$ в зависимости от скорости ветра для разных категорий устойчивости атмосферы $(\mathrm{A}, \mathrm{E}$, F), результаты расчетов приведены на рис. 5-11.

Найдено распределение по оси факела в приземном слое атмосферы отдельных составляющих выброса АЭС - концентраций РБГ, радионуклидов йода и аэрозолей. Определены соответствующие максимальные значения концентрации в приземном слое атмосферы отдельных составляющих выбросов, расстояние от устья трубы, на котором они достигаются. Определено также значение опасной скорости ветра и распределение концентраций РБГ, радионуклидов йода и аэрозолей в приземном слое атмосферы при этой скорости.

Расчеты проведены для следующих условий. Шероховатость подстилающей поверхности $\left(z_{0}\right)$ принята равной 40 см, температура выброса газоаэрозолей $\left(t_{0}\right)$ - равной $15{ }^{\circ} \mathrm{C}$, скорость выхода га- зов из устья вентиляционной трубы $\left(w_{0}\right)$ - равной $10 \mathrm{~m} / \mathrm{c}$. Температура окружающего атмосферного воздуха $(t)$ была принята в зависимости от категории состояния атмосферы в диапазоне от -20 до $+20{ }^{\circ} \mathrm{C}$.

Изображенные на рис. 5, 6 зависимости показывают, что фактором, наиболее влияющим на концентрацию в приземном слое атмосферы отдельных составляющих выброса атомной станции (РБГ, радионуклидов йода и аэрозолей), является категория состояния атмосферы (предельно неустойчивая $\mathrm{A}$, умеренно устойчивая $\mathrm{F}$, слабо устойчивая Е). Максимальное значение концентрации радионуклидов йода для категории Е при скорости ветра $2 \mathrm{~m} /$ с составляет $3 \cdot 10^{-4} \mathrm{Б \kappa} / \mathrm{m}^{3}$ (рис. $\left.6, a\right)$, что приблизительно в три раза меньше, чем при тех же условиях для категории состояния атмосферы А (рис. 5, а). Расстояние, на котором достигается максимум концентраций, также сильно зависит от категории: для $\mathrm{A}-1$ км, для $\mathrm{E}-4$ км.

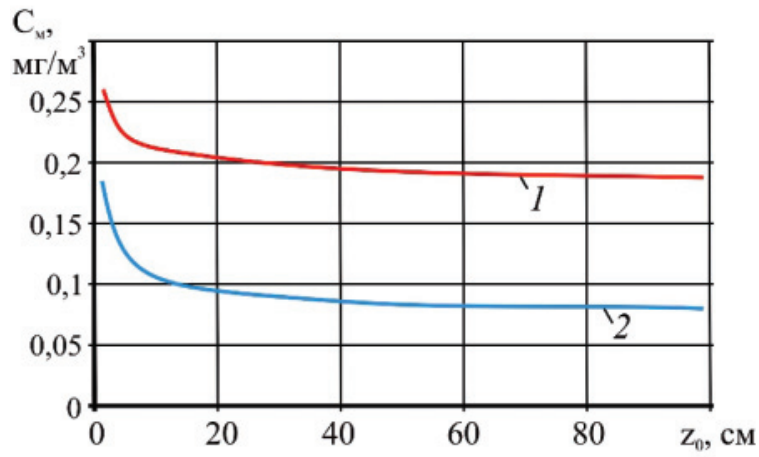

$a$

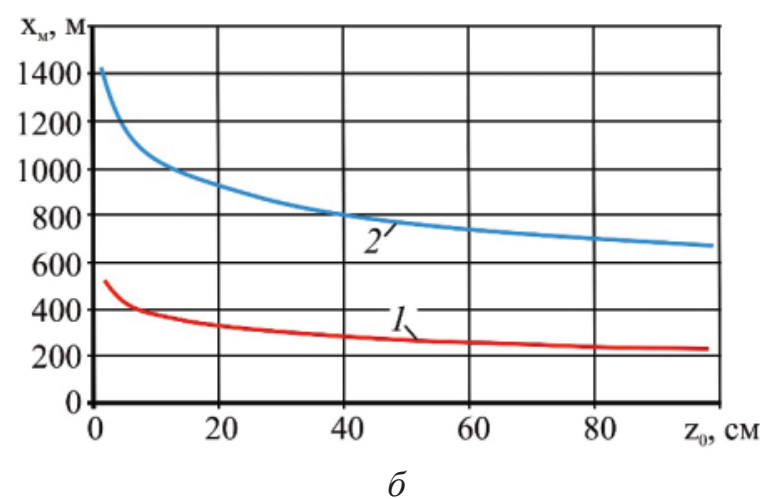

Puc. 4. Зависилость значений кониентрации $\mathrm{SO}_{2}, \mathrm{NO}_{2}($ a) и расстояния от источника (б) от шероховатости $\left(z_{0}\right)$ подстилающей поверхности при метеорологических условиях: 1 - неблагоприятных; 2 - благоприятных

Fig. 4. Dependence of $\mathrm{SO}_{2}, \mathrm{NO}_{2}$ concentration values (a) and the distance from the source $(\sigma)$ on the roughness $\left(z_{0}\right)$ of the underlying surface under meteorological conditions: 1 - adverse; 2-favorable

Скорость ветра наибольшее влияние оказывает на значение концентрации радионуклидов при категории Е состояния атмосферы. В диапазоне увеличения скорости ветра от 2 до $6 \mathrm{~m} /$ с максимальное значение концентрации падает в 2-3 раза (рис. 6). В то же время для категории А состояния 
атмосферы в том же диапазоне скорости ветра концентрация загрязняющих веществ падает не более чем в 1,5 раза (рис. 5$)$.

Очень существенно влияет значение скорости ветра на максимальную концентрацию йода при категории $\mathrm{F}$, что видно из рис. $7, a$. Максимальная концентрация йода падает более чем на порядок при росте скорости ветра от 0,2 до 5 м/с. Расстояние же, соответствующее максимальной концентрации, при этой категории существенно не меняется с изменением скорости ветра (рис. 7, б).
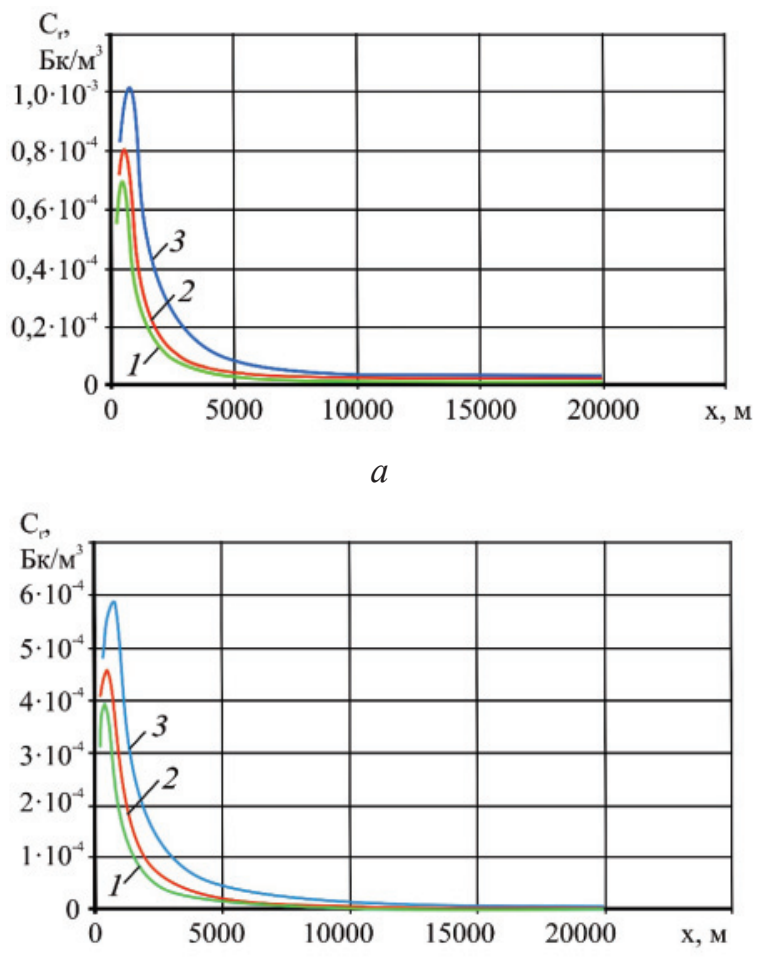

6

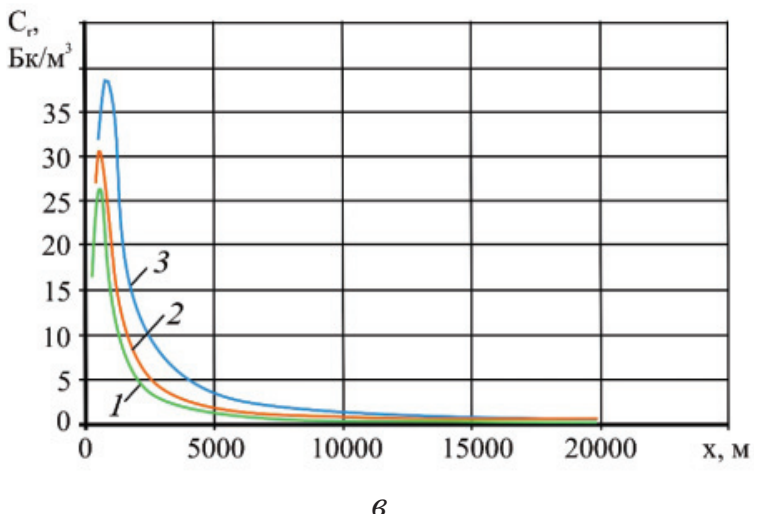

Pис.5. Распределение концентрации йода (а), аэрозолей (б) и РБГ (в) в приземном слое (категория $A, z_{0}=40$ см; $t=+15^{\circ} \mathrm{C} ; t_{0}=15^{\circ} \mathrm{C} ; w_{0}=10 \mathrm{~m} / \mathrm{c}$ ) при разных скоростях ве тра u: 1) 6 ; 2) 4 ; 3) $2 \mathrm{~m} / \mathrm{c}$

Fig. 5. Distribution of iodine (a), aerosols (б) and RNG (c) concen trations in the surface layer (category $A, z_{0}=40 \mathrm{~cm}$, $t=+15^{\circ} \mathrm{C} ; t_{0}=15^{\circ} \mathrm{C} ; w_{0}=10 \mathrm{~m} / \mathrm{s}$ ) at different wind speeds $u$ : 1) 6 ; 2) 4 ; 3) $2 \mathrm{~m} / \mathrm{s}$
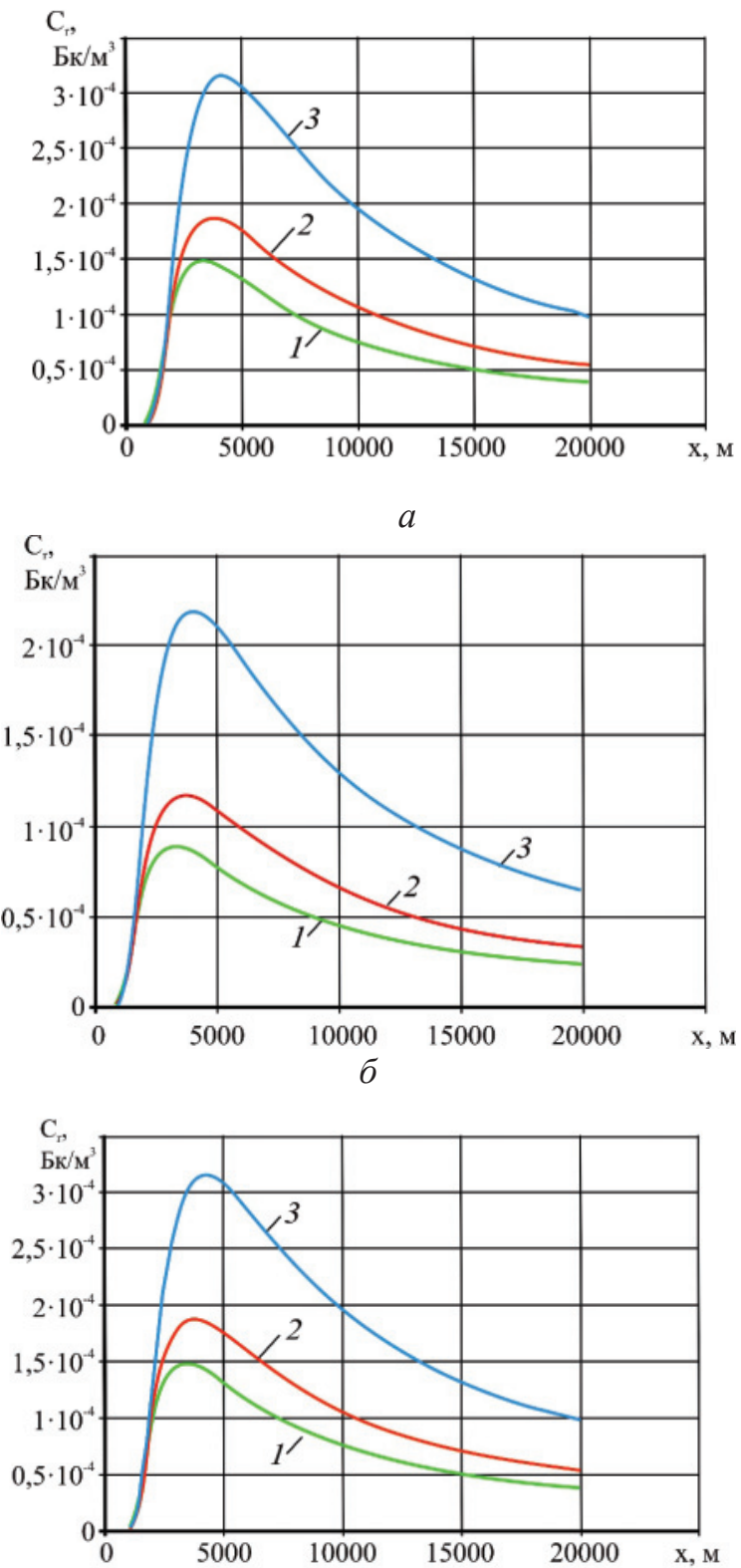

B

Pис.6. Распределение концентрации йода (а), аэрозолей (б) и РБГ (в) в приземном слое (категория $E, z_{0}=40$ см; $\left.t=+20^{\circ} \mathrm{C} ; t_{0}=15^{\circ} \mathrm{C} ; w_{0}=10 \mathrm{~m} / \mathrm{c}\right)$ при разных скоростях ветра $u: 1) 6$; 2) 4 ; 3) $2 \mathrm{~m} / \mathrm{c}$

Fig. 6. Distribution of iodine (a), aerosols (b) and RNG (c) concentration in the surface layer (category $E, z_{0}=40 \mathrm{~cm} ; t=+20^{\circ} \mathrm{C}$; $t_{0}=15^{\circ} \mathrm{C} ; w_{0}=10 \mathrm{~m} / \mathrm{s}$ ) at different wind speeds $u: 1$ ) 6 ; 2) 4; 3) $2 \mathrm{~m} / \mathrm{s}$

С использованием полученных данных для категории А при среднегодовом значении скорости ветра $4 \mathrm{~m} / \mathrm{c}$ построены концентрационные профили РБГ (рис. 9) и йода (рис. 10) по направлению ветра, на которые нанесены изолинии концентраций. По этим рисункам можно сказать, что след достаточно узкий, его ширина не превышает 400 м в самом широком месте (на расстоянии 300 м от трубы). Анализ данных показывает, что за преде- 
лами санитарно-защитной зоны (в радиусе 2-3 км от АЭС) концентрация радионуклидов становится пренебрежимо малой.

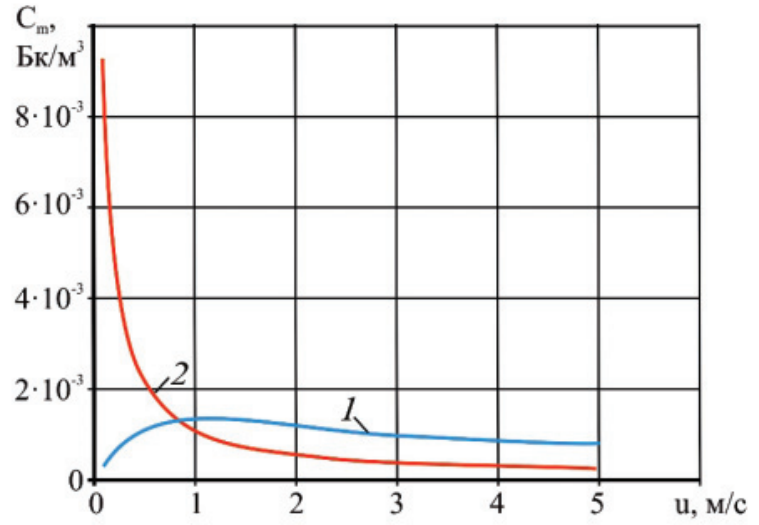

$a$

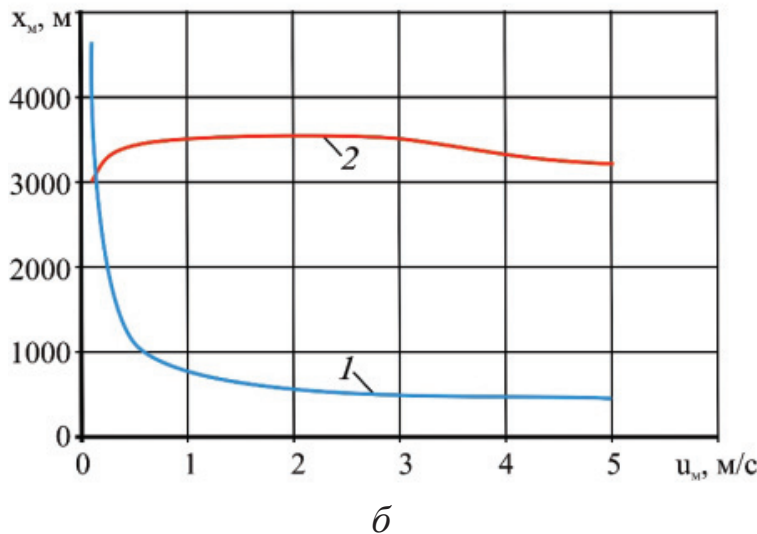

Pис.7. Зависимость максимальной концентрации йода в при земном слое (а), и расстояния от источника (б), на ко тором достигается максимальная концентращия, от скорости ветра и при разных категориях состояния ат мосферы $\left.z_{0}=40 \mathrm{~cm} ; t=+20^{\circ} \mathrm{C} ; t_{0}=15^{\circ} \mathrm{C} ; w_{0}=10 \mathrm{~m} / \mathrm{c}: 1\right)$ кате гория $A$; 2 ) категория $F$

Fig. 7. Dependence of the maximum iodine concentration in the surface layer (a), and the distance from the source (ס), where the maximum concentration is reached at different wind spe eds $u$ and atmospheric categories $z_{0}=40 \mathrm{~cm} ; t=+20^{\circ} \mathrm{C}$; $t_{0}=15^{\circ} \mathrm{C} ; w_{0}=10 \mathrm{~m} / \mathrm{s}: 1$ ) category $\mathrm{A} ; 2$ ) category $\mathrm{F}$

Сравнение значений концентраций в приземном слое атмосферы отдельных составляющих выброса АЭС с допустимыми концентрациями [12] показывает, что в рассмотренных условиях за пределами санитарно-защитной зоны концентрации РБГ, йода и аэрозолей заметно ниже допустимых значений. При этом надо иметь ввиду, что исходные данные соответствовали нормальной работе $\mathrm{AC}$ и был сделан ряд допущений, в частности, принято отсутствие фона активности.

\section{Выводы}

С целью повышения точности прогнозирования поведения выброса от энергообъектов в атмосфере проведен анализ математических моделей переноса загрязняющих веществ, рассмотрены алгоритмы и разработан программный комплекс для расчета рассеивания примесей в приземном слое атмосферы по двум методикам: МРР-2017 и Пасквилла.

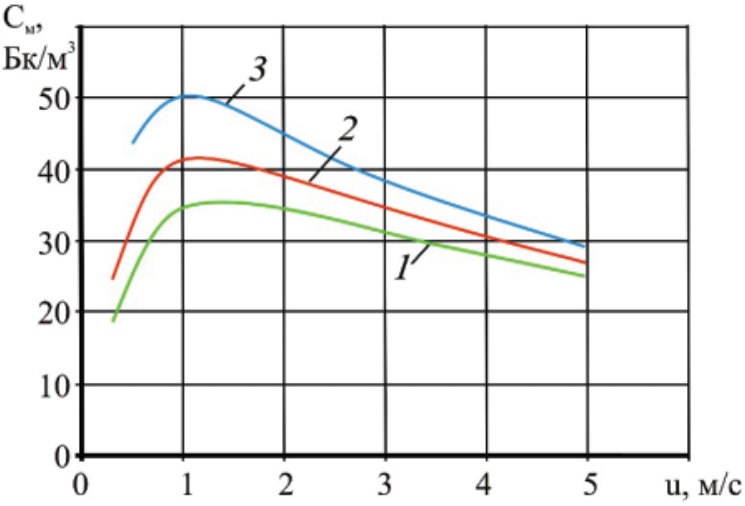

$a$

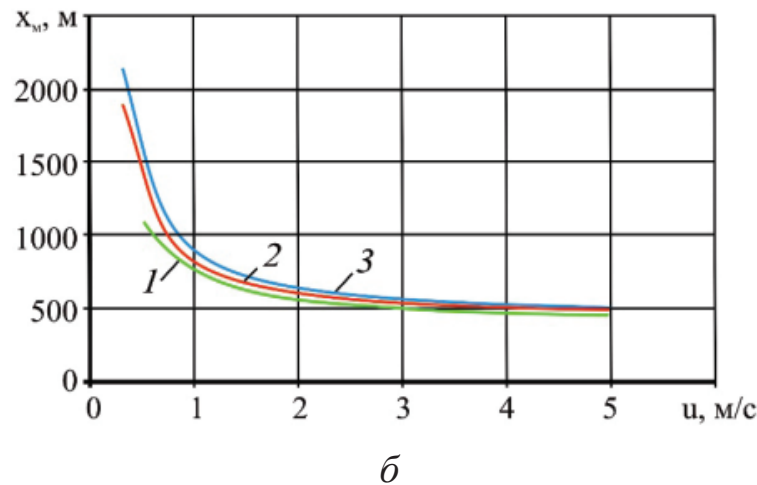

Pис. 8. Зависилость максилальной концентрации радиоактивных благородных газов в приземном слое (а) и расстояния от источника (б), соответствующего максимальной концентрации, от скорости ветра при разных значениях телпературы атмосрерного воздуха $t_{\mathrm{B}}$ (категория $\left.A ; z_{0}=40 \mathrm{~cm} ; t_{0}=15^{\circ} \mathrm{C} ; w_{0}=10 \mathrm{~m} / \mathrm{c}\right): 1$ ) $\left.t_{\mathrm{B}}=-20^{\circ} \mathrm{C} ; 2\right) t_{\mathrm{B}}=0^{\circ} \mathrm{C}$; 3) $t_{\mathrm{B}}=20^{\circ} \mathrm{C}$

Fig. 8. Dependence of the maximum concentration of radioactive noble gases in the surface layer ( $a$ ) and the distance from the source ( $\sigma$ ), corresponding to the maximum concentration, at different wind speeds and temperatures of atmospheric air $t_{\mathrm{a}}$ (category $A ; z_{0}=40 \mathrm{~cm} ; t_{0}=15^{\circ} \mathrm{C} ; w_{0}=10 \mathrm{~m} / \mathrm{s}$ ): 1) $t_{\mathrm{a}}=-20^{\circ} \mathrm{C}$; 2) $\left.t_{\mathrm{a}}=0^{\circ} \mathrm{C} ; 3\right) t_{\mathrm{a}}=20^{\circ} \mathrm{C}$

Сравнение значений концентраций в приземном слое атмосферы отдельных составляющих выброса АЭС с допустимыми концентрациями [12] показывает, что в рассмотренных условиях за пределами санитарно-защитной зоны концентрации РБГ, йода и аэрозолей заметно ниже допустимых значений. При этом надо иметь ввиду, что исходные данные соответствовали нормальной работе $\mathrm{AC}$ и был сделан ряд допущений, в частности, принято отсутствие фона активности.

\section{Выводы}

С целью повышения точности прогнозирования поведения выброса от энергообъектов в атмосфере проведен анализ математических моделей переноса загрязняющих веществ, рассмотрены алгоритмы и 


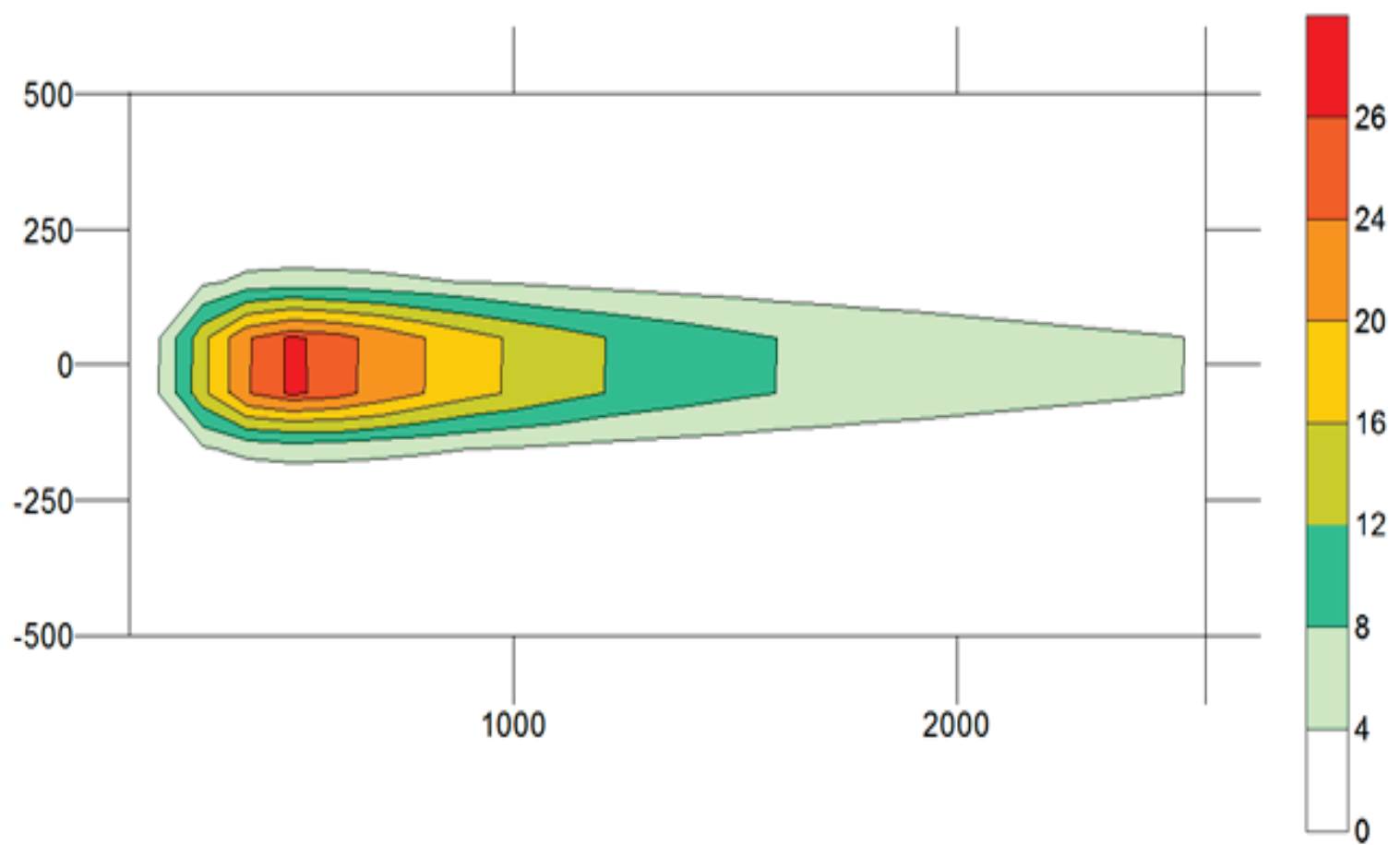

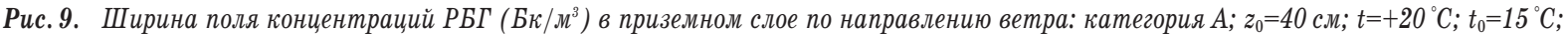
$w_{0}=10 \mathrm{~m} / c ; u=4 \mathrm{~m} / \mathrm{c}$

Fig. 9. Width of the field of radioactive noble gases concentrations $\left(B q / \mathrm{m}^{3}\right)$ in the surface layer in the direction of the wind: category A; $z_{0}=40 \mathrm{~cm} ; t=+20^{\circ} \mathrm{C} ; t_{0}=15^{\circ} \mathrm{C} ; w_{0}=10 \mathrm{~m} / \mathrm{s} ; u=4 \mathrm{~m} / \mathrm{s}$

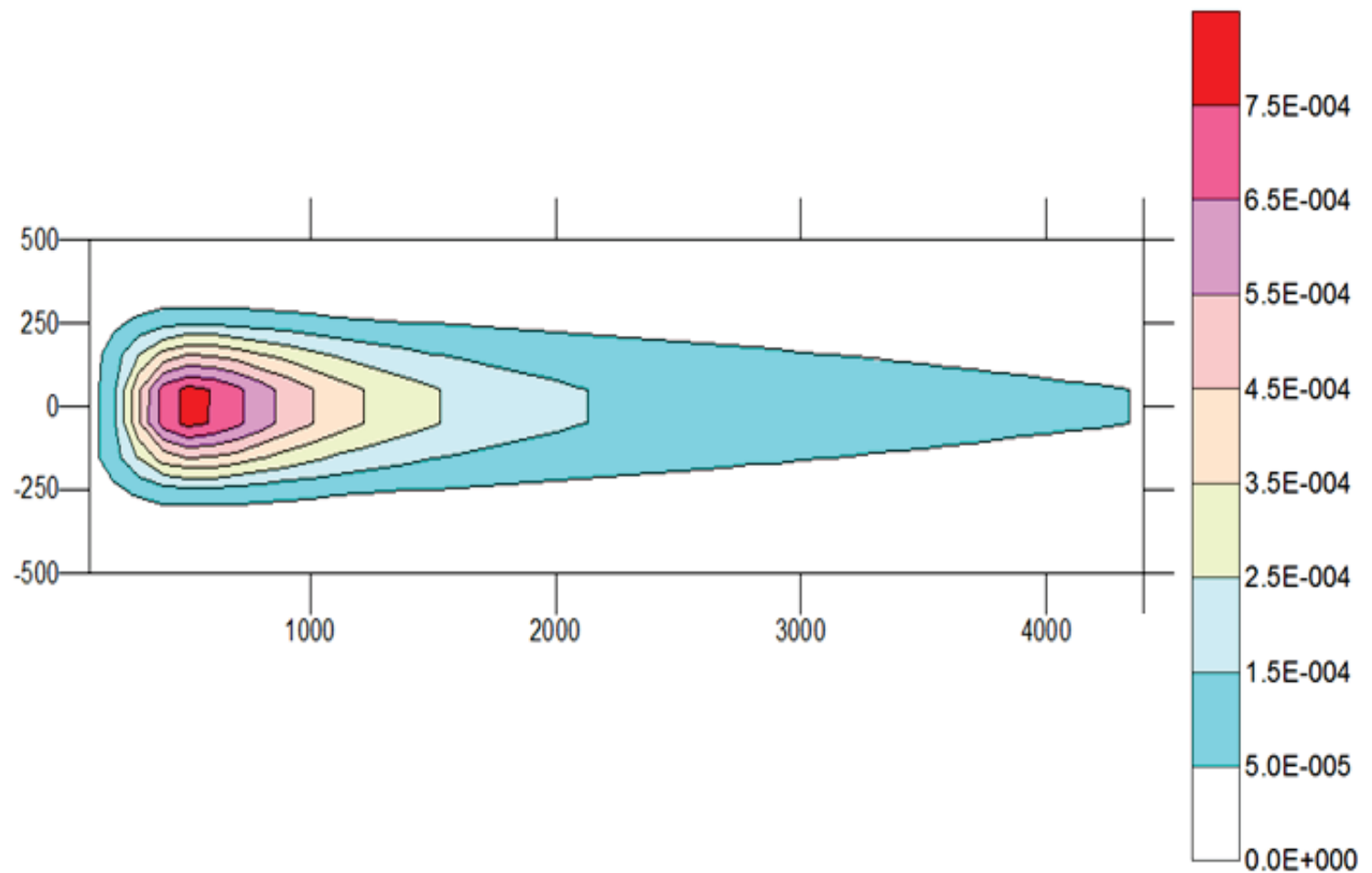

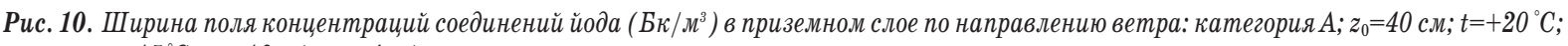
$t_{0}=15^{\circ} \mathrm{C} ; w_{0}=10 \mathrm{~m} / \mathrm{c} ; u=4 \mathrm{~m} / \mathrm{c}$

Fig. 10. Width of concentrations field of iodine compounds $\left(\mathrm{Bq} / \mathrm{m}^{3}\right)$ in the surface layer wind direction: category $A ; z_{0}=40 \mathrm{~cm} ; t=+20^{\circ} \mathrm{C}$; $t_{0}=15^{\circ} \mathrm{C} ; w_{0}=10 \mathrm{~m} / \mathrm{s} ; u=4 \mathrm{~m} / \mathrm{s}$ 
разработан программный комплекс для расчета рассеивания примесей в приземном слое атмосферы по двум методикам: МРР-2017 и Пасквилла.

По результатам исследования сделан вывод о том, что для расчетов рассеивания газообразных загрязняющих веществ от непрерывно действующего источника в условиях постоянства метеорологических факторов предпочтительнее использовать методику Пасквилла, так как она позволяет учесть более широкий набор определяющих параметров, адекватно описывающих местные метеорологические условия и тип подстилающей поверхности.

Разработанный программный комплекс «SKAT» может быть использован для прогнозирования приземных концентраций загрязняющих веществ и расчетов рассеивания загрязняющих веществ в атмосферном воздухе при решении задач мониторинга выбросов ЗВ в условиях действующих объектов.

Результаты расчетных исследований, выполненных с помощью принятых моделей, показыва-

\section{СПИСОК ЛИТЕРАТУРЫ}

1. Wark K., Warner C.F., Davis W.T. Air Pollution. Its Origin and Control. $3^{\text {rd }}$ ed. - Menlo Park: Addison Wesley Longman Inc., 1998. $-573 \mathrm{p}$.

2. Положение о повышении точности прогностических оценок радиационных характеристик радиоактивного загрязнения окружающей среды и дозовых нагрузок на персонал и население. Утв. приказом Федеральной службы по экологическому, технологическому и атомному надзору от 8 июня 2010 г. № 465. - М.: Ростехнадзор, 2010. - 111 с.

3. Методы расчетов рассеивания вредных (загрязняющих) веществ в атмосферном воздухе. Утв. Приказом Минприроды России от 06.06.2017 г. № 273. - М.: Ростехнадзор, 2017. $110 \mathrm{c.}$

4. Индикация компонентами природной среды трансграничного переноса загрязняющих веществ на территорию горного Алтая / Ю.В. Робертус, В.Н. Удачин, Л.П. Рихванов, Д.В. Юсупов, А.В. Кивацкая, Р.В. Любимов // Известия Томского политехнического университета. Инжиниринг георесурсов. - 2016. T. 327. - № 9. - С. 39-48,

5. Илюшин Б.Б., Митин И.В., Сиковский Д.Ф. Модели функции плотности вероятностей для описания распространения примеси в конвективном пограничном слое атмосферы // Известия Томского политехнического университета. Инжиниринг георесурсов. - 2015. - Т. 326. - № 7. - С. 51-59.

6. Оценка влияния метеорологических и орографических условий на аэрозольное загрязнение снежного покрова на юге Томского региона / Б.Д. Белан, В.С. Бучельников, В.Ф. Лысова, Д.В. Симоненков, А.В. Таловская, М.П. Тентюков, Е.Г. Язиков // Оптика атмосферы и океана. - 2018. - Т. 31. - № 6. C. $492-500$.

7. Characterization of solid airborne particles deposited in snow in the vicinity of urban fossil fuel thermal power plant (Western Siberia) / A.V. Talovskaya, E.G. Yazikov, E.A. Filimonenko, J.-C. Lata, J. Kim, T.S. Shakhova / / Environmental Technology. 17 September 2018. - V 39. - Iss. 18. - P. 2288-2303.

8. Fluorine concentration in snow cover within the impact area of aluminium production plant (Krasnoyarsk city) and coal and gasfired power plant (Tomsk city) / A.V. Talovskaya, N.A. Osipova, S.A. Polikanova, N.P. Samokhina, E.A. Filimonenko, E.G. Yazikov, I.A. Matveenko // IOP Conference Series: Earth and Environmental Science. - 2015 - V. 27. - P. 012043-1-012043-6. ют, что основными определяющими факторами являются: состояние атмосферы и температура атмосферного воздуха. Менее заметное влияние оказывает наличие осадков.

С использованием программного комплекса «SKAT» на основе метода Пасквилла выполнены исследования поведения концентраций радионуклидов в приземном слое атмосферы в условиях г. Северска Томской области для проектируемого энергоблока АЭС с реактором БРЕСТ-300 и пристанционным ядерным топливным циклом.

Проведены расчеты концентраций радионуклидов выброса РБГ, йода и аэрозолей в зависимости от скорости ветра для разных категорий устойчивости атмосферы. На основании полученных данных построены графики распределения концентраций и поля концентрации радионуклидов по направлению ветра, анализ которых нанесены изолинии концентраций. Анализ профилей показывает, что за пределами санитарно-защитной зоны значение концентрации радионуклидов невелико.

9. Duynkerke P.G. Application of the E turbulence closure model to the neutral and stable atmospheric boundary layer // Journal of the Atmospheric Sciences. - 1988. - V. 45. - № 5. - P. 865-880.

10. Arya S. Pal Air Pollution Meteorology and Dispersion. $1^{\text {st }}$ ed. Oxford: Oxford University Press. 1998. - 320 p.

11. Mandurino C., Vestrucci P. Using meteorological data to model pollutant dispersion in the atmosphere // Environmental Modelling \& Software. - 2009. - V. 24. - Iss. 2. - P. 270-278.

12. Допустимые выбросы радиоактивных и химических веществ в атмосферу / под ред. Е.Н. Теверовского, И.А. Терновского. М.: Энергоатомиздат, 1985. - 216 с.

13. Safety assessment for facilities and activities. International Atomic Energy Agency Safety Standards Series No. GSR. Part 4 (Rev. 1). Vienna, IAEA, 2016.63 p.

14. Fractional derivative models for atmospheric dispersion of pollutants / A.G.O. Goulart, M.J. Lazo, J.M.S. Suarez, D.M. Moreira // Physica A: Statistical Mechanics and its Applications. - 2017. V. 477. - P. 9-19.

15. Илюшин Б.Б., Курбацкий А.Ф. Моделирование распространения примеси в конвективном пограничном слое атмоссеры // Известия РАН. Физика атмосферы и океана. - 1996. - Т. 32. № 3. - C. 307-322.

16. Методика разработки и установления нормативов предельно допустимых выбросов радиоактивных веществ в атмосферный воздух. Утв. приказом Ростехнадзора от 07.11.2012 г. № 639.

17. Берлянд М.Е. Прогноз и регулирование загрязнений атмосферы. - Л.: Гидрометеоиздат, 1985. - 272 с.

18. Van Dop H., Frans T.M. Nieuwstadt // Atmospheric Turbulence and Air Pollution Modelling. - Dordrecht: D. Reider Publishing Company, 1981. $-358 \mathrm{p}$.

19. Encyclopedia of Atmospheric Sciences. $2^{\text {nd }}$ ed. Eds: J.A. Curry, J.A. Pyle. - London: Academic Press, 2015. - 2998 p.

20. The comparison of Lagrangian and Gaussian models in predicting of air pollution emission using experimental study, a case study: Ammonia emission / M. Asadi, G. Asadollahfardi, H. Fakhraee, M. Mirmohammadi // Environmental Modeling \& Assessment. 2017. - V. 22. - P. 27-36.

21. Pasquill F., Smith F.B. Atmospheric diffusion. $3^{\text {rd }}$ ed. - Chichester: Ellis Horwood Ltd., 1983. -429 p.

Поступила 04.06.2019 2. 


\section{Информация об авторах}

Антонова A.M., кандидат технических наук, доцент научно-образовательного центра И.Н. Бутакова Инженерной школы энергетики Национального исследовательского Томского политехнического университета.

Воробъев A.B., кандидат технических наук, доцент научно-образовательного центра И.Н. Бутакова Инженерной школы энергетики Национального исследовательского Томского политехнического университета.

Воробъев В.A., инженер научно-образовательного центра И.Н. Бутакова Инженерной школы энергетики Национального исследовательского Томского политехнического университета.

Дутова E.M., доктор геолого-минералогических наук, профессор отделения геологии Инженерной школы природных ресурсов Национального исследовательского Томского политехнического университета.

Покровский В.Д., кандидат геолого-минералогических наук, старший научный сотрудник Геолого-географического факультета Национального исследовательского Томского государственного университета. 
UDC 628.58

\title{
MODELLING DISTRIBUTION OF CONTAMINATING SUBSTANCES OF ELECTRIC POWER EMISSIONS IN THE ATMOSPHERE ON THE BASIS OF THE SKAT PROGRAMMING COMPLEX
}

\author{
Aleksandra M. Antonova', \\ anton@tpu.ru \\ Aleksandr V. Vorobev',
worob@tpu.ru \\ Vladimir A. Vorobev', \\ worob@tpu.ru \\ Ekaterina M. Dutova', \\ dutova@tpu.ru \\ Vitaly D. Pokrovskiy, \\ pokrovskiy.v@gmail.com \\ 1 National Research Tomsk Polytechnic University, \\ 30, Lenin Avenue, Tomsk, 634050, Russia. \\ ${ }^{2}$ National Research Tomsk State University, \\ 36, Lenin Avenue, Tomsk, 634050, Russia.
}

Relevance of the research lies in the necessity of accuracy improvement of independent assessments at forecasting the content of pollutants in the air emitted by energy facilities, including nuclear power plants.

The aim of the research is justifying the choice of a method for calculating the gaseous pollutant distribution from continuously operating sources located at high altitude with consideration of meteorological factors and operation test of the software package. Objects of the research are the method of calculating the dispersion of gaseous pollutants MRR-2017 and the Pasquill method for calculating pollution from a continuously operating high source under various steady meteorological conditions.

Methods: simulation and computational analysis based on the developed computer software complex "SKAT».

Results. Methods for dispersion of gaseous pollutants in the atmosphere: MRR-2017 and Pasquill were analyzed. It was concluded that the Pasquill method based on the Gauss model is preferable for calculating the distribution of gaseous pollutants emitted by energy facilities at various atmospheric conditions of steady meteorological factors. The developed software package "SKAT» can be used to predict pollutant concentration values near the earth surface and calculate dispersion of pollutants in the atmospheric air when solving the problems of monitoring emissions of pollutants in the presence of currently operating enterprises. Using the Gauss model the authors have studied the distribution of the emitted radionuclides concentration in the atmospheric layer next to the earth surface coursed by the designed power unit with the BREST-300 reactor and the on-site nuclear fuel cycle for conditions of Seversk city, Tomsk Region.

Key words:

Pollutants, modeling, dispersion, atmospheric air, turbulent diffusion, environmental protection.

\section{REFERENCES}

1. Wark K., Warner C.F.\& Davis W.T. Air Pollution. Its Origin and Con trol. $3^{\text {rd }}$ ed. Menlo Park, Addison Wesley Longman Inc., 1998. 573 p.

2. Polozhenie o povyshenii tochnosti prognosticheskikh otsenok radiatsionnykh kharakteristik radioaktionogo zagryazneniya okruzhayushchey sredy i dozovykh nagruzok na personal i nasele nie [The provision on improving the accuracy of prognostic estimates of the radiation characteristics of radioactive contamination of the environment and dose loads on personnel and the public]. Utv. prikazom Federalnoy sluzhby po ekologicheskomu, tekhnologicheskomu i atomnomu nadzoru ot 8 iyunya $2010 \mathrm{~g}$. № 465 [Approved by the Order of the Federal Service for Environmental, Technological and Nuclear Supervision dated June 8, 2010 No. 465]. Moscow, Rostekhnadzor Publ., 2010. 111 p.

3. Metody raschetov rasseivaniya vrednykh (zagryaznyayushchikh) veshchestv $v$ atmosfernom vozdukhe [Calculation methods for dispersion of harmful (polluting) substances in the atmospheric air]. Utv. Prikazom Minprirody Rossii ot 06.06.2017 g. № 273 [Approved by the order of the Ministry of Natural Resources of Russia of 06.06.2017, No. 273]. Moscow, Rostekhnadzor Publ., $2017.110 \mathrm{p}$.
4. Robertus Yu.V., Udachin V.N., Rikhvanov L.P., Yusupov D.V., Kivatskya A.V., Lyubimov R.V. Indication by components of the natural environment of transboundary transport of pollutants to the territory of the mountainous Altai]. Bulletin of the Tomsk Polytechnic University. Geo Assets Engineering, 2016, vol. 327, no. 9, pp. 39-48. In Rus.

5. Ilyushin B.B., Mitin I.V., Sikovskii D.F. Models of probability density function for describing pollutant distribution in convective planetary boundary layer. Bulletin of the Tomsk Polytechnic University. Geo Assets Engineering, 2015, vol. 326, no. 7, pp. 51-59. In Rus.

6. Belan B.D., Buchelnikov V.S., Lysova V.F., Simonenkov D.V., Talovskaya A.V., Tentyukov M.P. and Yazikov E. G. Estimation of the Effect of Meteorological and Orographic Conditions on Aerosol Contamination of the Snow Cover in the South of Tomsk Region. Atmospheric and Oceanic Optics, 2018, vol. 31. no. 06. pp. 656-664.

7. Talovskaya A.V., Yazikov E.G., Filimonenko E.A., Lata J.-C, Kim J., Shakhova T.S. Characterization of solid airborne particles deposited in snow in the vicinity of urban fossil fuel thermal power plant (Western Siberia). Environmental Technology, 2018, vol. 39, Iss. 18, pp. 2288-2303. 
8. Talovskaya A.V., Osipova N.A., Polikanova S.A., Samokhina N.P., Filimonenko E.A., Yazikov E.G., Matveenko I.A. Fluorine concentration in snow cover within the impact area of aluminium production plant (Krasnoyarsk city) and coal and gas-fired power plant (Tomsk city). IOP Conference Series: Earth and Environmental Science, 2015, vol. 27, pp. 012043-1-012043-6.

9. Duynkerke P.G. Application of the E turbulence closure model to the neutral and stable atmospheric boundary layer. Journal of the Atmospheric Sciences, 1988, vol. 45, no. 5, pp. 865-880.

10. Arya S. Pal Air Pollution Meteorology and Dispersion. $1^{\text {st }}$ ed. Oxford, Oxford University Press. 1998. 320 p.

11. Mandurino C., Vestrucci P. Using meteorological data to model pollutant dispersion in the atmosphere. Environmental Model ling \& Software, 2009, vol. 24, Iss. 2, pp. 270-278.

12. Dopustimye vybrosy radioaktivnykh $i$ khimicheskikh veshchestv $v$ atmosferu [Permissible emissions of radioactive and chemical substances into the atmosphere]. Eds. E.N. Teverovsky, I.A. Ternovsky. Moscow, Energoatomizdat Publ., 1985. 216 p.

13. Safety assessment for facilities and activities. International Atomic Energy Agency Safety Standards Series No. GSR. Part 4 (Rev. 1). Vienna, IAEA, 2016. $63 \mathrm{p}$.

14. Goulart A.G.O., Lazo M.J., Suarez J.M.S., Moreira D.M. Fractional derivative models for atmospheric dispersion of pollutants. Physica A: Statistical Mechanics and its Applications, 2017, vol. 477, pp. 9-19.

15. Ilyushin B.B., Kurbatskii A.F. Modelirovanie rasprostraneniya primesi v konvektivnom pogranichnom sloe atmosfery [Simula-

Information about the authors

Aleksandra M. Antonova, Cand. Sc., assistant professor, National Research Tomsk Polytechnic University.

Aleksandr V. Vorobev, Cand. Sc., assistant professor, National Research Tomsk Polytechnic University.

Vladimir A. Vorobev, engineer, National Research Tomsk Polytechnic University.

Ekaterina M. Dutova, Dr. Sc., professor, National Research Tomsk Polytechnic University.

Vitaly D. Pokrovskiy, Cand. Sc., assistant professor, National Research Tomsk State University. tion of distribution of impurities in the convective atmospheric boundary layer]. Izvestiya Rossiiskoi akademii nauk. Fizika atmosfery i okeana, 1996, vol. 32, no. 3, pp. 307-322.

16. Metodika razrabotki i ustanovleniya normativov predelno dopustimykh vybrosov radioaktivnykh veshchestv $v$ atmosferny vozdukh [Methods of developing and establishing standards for maximum permissible emissions of radioactive substances into the air]. Utv. prikazom Rostekhnadzora ot 07.11.2012 g. № 639 [Approved by the Order of Rostekhnadzor dated 07.11.2012, No. 639].

17. Berlyand M.E. Prognoz i regulirovanie zagryazhenii atmosfery [Prediction and regulation of air pollution]. Leningrad, Gidrometeoizdat Publ., 1985. $272 \mathrm{p}$.

18. Van Dop H., Frans T.M. Nieuwstadt. Atmospheric Turbulence and Air Pollution Modelling. Dordrecht, D. Reider Publishing Company, 1981. - $358 \mathrm{p}$.

19. Encyclopedia of Atmospheric Sciences. $2^{\text {nd }}$ ed. Eds: J.A. Curry, J.A. Pyle. London, Academic Press, 2015. 2998 p.

20. Asadi M., Asadollahfardi G., Fakhraee H., Mirmohammadi M. The comparison of Lagrangian and Gaussian models in predicting of air pollution emission using experimental study, a case study: Ammonia emission, Environmental Modeling \& Assessment, 2017, vol. 22, pp. 27-36.

1. Pasquill F., Smith F.B. Atmospheric diffusion. $3^{\text {rd }}$ ed. Chichester, Ellis Horwood Ltd., 1983. 429 p.

Received: 4 June 2019. 